\title{
ESTUDIO CUALITATIVO DEL FITOPLANCTON DE LA LAGUNA ALTOANDINA PAUCARANI-TACNA
}

\author{
Juan Franco L. ${ }^{1}$ \\ Victorino Delgado T. ${ }^{2}$ \\ Liduvina Sulca Q. ${ }^{3}$
}

\section{RESUMEN}

Se estudio y analizó el fitoplancton de la laguna Paucarani de Tacna. Ambiente lacustre de la Cordillera del Sur, colindante con los países de Chile y Bolivia. El análisis comprendió un lapso de 7 meses (enero-julio 1996). Este cuerpo de agua caracterizado por presentar cierta dureza en su composición química no es aprovechado por el poblador andino como un medio de actividad piscícola, 10 que de alguna manera podría desarrollar más las fronteras vivas de la Región.

Se registraron especies pertenecientes a las divisiones Cyanophyta, Euglenophyta, Dinophyta, Bacillariophyta y Chlorophyta, que se distribuyeron en términos de dominancia de la siguiente manera: $25,82 \%, 9,68 \%, 3,2 \%, 54,42 \%$ y $6,45 \%$, respectivamente.

Se constituye la más abundante en diversidad de especies, la familia Bacillariophyceae.

\section{A B S T R A C T}

The Plankton of the Paucasani lagoon in Tacna w:s analyzed and studied. It is a lake environment in the southern higitionds contiguous with Chile and Bolivia. The Analysis lasted 7 months (January - July 1996). A characteristic of this woter es its hardress in chemical composition. The local residents don't take advantage of this is a means of Piscicota activity, but this could help to develop the living borders of the region.

Species that belong to Division Cyanophyta, Euglenophyta, Dinophyta, Bacillariophyta and Chlorophyta were examined. They were classified according to the dominant spicies in the followinj way: $25,8 \%$; $9,68 \% ; 3,2 \% ; 54,42 \% ;$ y $6,45 \%$ respectively.

The most abundant spicies was the Bacillariophyceae family. 


\section{Ciencia \& Desarrollo}

\section{INTRODUCCIÓN}

La Cordillera de los Andes en el sur del Perú contiene un número singular de ecosistemas acuáticos: lagos, lagunillas, ríos, etc. Con una vegetación típica de la zona.

El estudio de fitoplancton de un medio lacustre permite conocer la estructura de la producción biológica, la misma que está compuesta por organismos que se distribuyen en lagos de composición química variada, desarrollando cada uno de ellos, una función específica dentro de la ecología del medio.

El presente trabajo se presenta como un aporte al conocimiento del tipo de fitoplancton que se halla en estos cuerpos de aguas altoandinos, con el propósito de potencializar su utilidad en la crianza de truchas en jaulas flotantes, como una forma de reactivar las fronteras vivas de nuestra zona, mediante los pobladores del lugar; los que únicamente están dedicados a la crianza de camélidos sudamericanos.

En este contexto, el objetivo principal del trabajo es el de proporcionar una información taxonómica de los grupos que conforman la estructura de fitoplancton, como también dar a conocer las principales caractetísticas limnológicas del medio.

\section{MATERIAL Y MÉTODOS}

La zona de estudio comprende la laguna de Paucarani, ubicada en los alrededores de la localidad de Alto Perú, departamento de Tacna, cerca al área limítrofe con el país de Chile. (Fig. №1)

El cuerpo de agua se halla comprendido entre las coordenadas geográficas: $16^{\circ} 58^{\prime}$ Lat. S. y $70^{\circ} 06^{\prime}$ Long. 0. a 4,135 m.s.m.m. (Fig. $\mathrm{N}^{\circ} 2$ )

El lugar de trabajo está caracterizado principalmente por ocomales y gramíneas. El área pantanosa que rodea al lago, alterna con charcos pequeños; permitiendo que diversas aves puedan anidar y descansar; encontrando un hábitat apropiado para su reproducción.

Las colecciones (30 muestras) se analizaron entre los meses de enero a julio 1996, siguiendo la metodología estandarizada para el estudio de fitoplancton, empleando una red de 60 y 80 um. de cocada. El análisis morfométrico de las especies fue realizada en el Laboratorio de botánica, utilizando un microscopio compuesto binocular con cámara fotográfica incorporada. La identificación de especies es realizada mediante técnicas de lavado y bibliografía especializada, como Prescott (1978), Manguin (1966), Fernández (1984), Montoya (1993), etc.

Los análisis físico-químicos del agua se registran en el Anexo $N^{\circ} 1$.

\section{RESULTADOS Y DISCUSIÓN}

Producto del análisis de la microflora se presentan diferentes grupos de organismos fotosintéticos, de los que se describe su ubicación taxonómica y características ecológicas.

La división Cyanophyta está respresentada por 3 géneros y 4 especies; la división Euglenophyta representada por 1 género y 2 especies; la división Dinophyta incluye un solo género; la división Bacillariophyta representada por 3 géneros y 14 especies y la división Chlorophyta, representada por 3 especies.

La familia Bacillariophyceae se caracteriza por sus predominancia absoluta, sobre el resto de familias de las diferentes divisiones. Esto difiere en algo con lo reportado por Montoya (1993), que incluía predominancia de Chlorophytas para lagunas altoandinas de nuestro país. Los géneros Synedra, Entomoneis y Amphora se presentan como los más abundantes dentro de las diatomeas, tipificando que las aguas del lago Paucarani son duras (Cole 1988).

La división Cyanophyta esta representada, por Nostoc commune como la especie dominante del grupo, siendo utilizada por los pobladores altoandinos como alimento y para comercialización, tal como ocurre y para otras zonas de la serranía del Perú (Aldave, 1989).

La división Euglenophyta incluye 3 representantes, de los cuales 2 comprenden al género Euglena y 1 a Trechelomona, siendo este ultimo colectado en áreas pantanosas que de alguna manera caracterizan medios ricos en hierro (Fernández, 1987). Coincidiendo en esto con Montoya (1993), que reporta que este organismo se desarrolla en medios cargados de hierro y manganeso.

La división Dinophyta incluye únicamente al género Gymnodinium, correspondiendo a un dinoflagelado desnudo (no tecado). Su presencia en el medio analizado es muy escasa, se discute aún, su importancia en aguas continentales.

La división Chlorophyta está representada escasamente por 3 especies, probablemente por las condiciones del medio. Predomina el género Ulothrix, sobre el resto de clorofitas, esto posiblemente puede deberse a que las aguas del lago sean ligeramente duras, permitiendo el desarrollo de diatomeas y cianofitas principalmente (Palmer, 1962).

\section{Reseña Taxonómica}

CYANOPHYTA

CYANOPHYCEAE

O. Chroococcales

Chroococcaceae

Agmenellum quadruplicatum Drouet. 
(Fig. 1)

O. Hormogonales

Oscillatoriaceae

Oscillatoria curviceps Ghose.

(Fig. 2)

Oscillatoria subuliformis Kutz.

(Fig. 3)

Phormidium sp Menegh.

(Fig. 4)

Nostocaceae

Nostoc commune Vaucher.

(Fig. 5)

Nostoc sp. Vaucher.

(Fig. 6)

Anabaena constricta Koppe.

(Fig. 7)

Anabaena sp. Logula.

(Fig. 8)

\section{EUGLENOPHYTA}

EUGLENOPHYCEAE (9,6\%)

O. Euglenales

Euglenaceae

Euglena elongata

(Fig. 9)

Euglena acus Ehrenbergii.

(Fig. 10)

Trachelomona sp. Stein.

(Fig. 11)

DINOPHYTA

DINOPHYCEAE (3,2\%)

0 . Gymnodiniales

Gymnodiniaceae

Gymnodinium sp. Stein

(Fig. 12)

BACILLARIOPHYTA

BACILLARIOPHYCEAE $(54,8 \%)$

O.Fragilariales

Fragilariaceae

Fragilaria capucina Lyngbye

(Fig. 13)

Synedra pseudogoulardii Ehrenberg

(Fig. 14)
Synedra nana Meist

(Fig. 15)

Synedra sp. Ehren

(Fig. 16)

O. Naviculales

Naviculaceae

Navicula mutica Bory

Fig. 17

Diploneis subovalis Bory

(Fig. 18)

Anomoeneis sphaerophora Bory

(Fig. 19)

Entomoneidaceae

Entomoneis alata Ehrem

(Fig. 20)

Entomoneis sp. Ehrem

(Fig. 21)

Cymbellaceae

Cymbella pseudonorvejica Agardh

(Fig. 22)

Gomphonemaceae

Gomphonema parvulum Ehrenberg

(Fig. 23)

O. EPITHEMIALES

Epithemiaceae

Rhopalodia gibberula Ehrenberg

(Fig. 24)

O. ACHANTHALES

Achnanthaceae

Rhoiscospheria curvata Granow

(Fig. 25)

O. NITZSCHIALES

Nitzschiaceae

Nitzschia molesto Manguin (6,45\%)

(Fig. 26)

Nitzschia bacata Massall

(Fig. 27)

Nitzschia peruviana Manguin

(Fig. 28)

Nitzschia sp. Hassall

(Fig. 29)

CHLOROPHYTA

Chlorophyceae

O. Chlorococcales 


\section{HYDRODICTYACEAE}

Pediastrum boryanum Menegluini

(Fig. 30)

O.ULOTHRICHALES

Ulothrichaceae

Ulothrix cylindricus Prescott

(Fig. 31)

\section{CONCLUSIONES}

Las especies y géneros que representan al fitoplancton altoandino de la laguna Paucarani del dpto. de Tacna, ubicado aproximadamente a 4,150 m.s.n.m., corresponden a un total de 31 especies distribuidas en los siguientes grupos: Cyanophyta con 8 representantes; Euglenophyta con 3 representantes; Dinophyta únicamente con un representante; los Bacillariophyta con 17 representantes y los Chlorophyta con 2 representantes.

De lo anterior se observa una dominancia de las Bacillariophyta dentro de la estructura del fitoplancton, caracterizando un posible medio de aguas duras, sin embargo, se toma en cuenta que las diatomeas pueden tener su afloración en estaciones de otoño, a medida que las aguas superficiales se enfrían y la desaparición de la termoclina permite la mezcla de las aguas superficiales con aquellas más profundas y ricas, en nutrientes (Happy-Wood 1976).

Las Cyanophyta se constituyen en el segundo grupo mejor representado en términos de diversidad de organismos. La especie Nostoc commune se presenta en alta biomasa entre los meses de diciembre a marzo, epoca en que los pobladores andinos la colectan para su consumo y comercialización.
ANEXO N¹ Análisis físico-químico: lago Paucarani-Tacna 1996

\begin{tabular}{|l|r|r|}
\hline Parámetro & Zona I & Zona II \\
\hline Temperatura $\left({ }^{\circ} \mathrm{C}\right)$ & 10 & 10 \\
$\mathrm{pH}$ & 7.9 & 7.8 \\
Transparencia $(\mathrm{cm})$ & 14 & 13 \\
Conductividad $(\mathrm{ms} / \mathrm{cm})$ & 200 & 216 \\
Calcio (mg/l) & 4.92 & 05.2 \\
Dureza total $\left(\mathrm{mg} / \mathrm{ICO}_{3} \mathrm{Ca}\right)$ & 100 & 108 \\
OD (mg/l) & 11,29 & 11,56 \\
Ortofosfatos $(\mathrm{mg} / \mathrm{l})$ & 0,71 & 0,73 \\
$\mathrm{CO}_{2}$ (mg/l) & 1,5 & 1,7 \\
\end{tabular}

Análisis realizados en Lab. de química U.N.J.B.G.

Los Euglenophytos se hallan representados por pocos organismos, su presencia tiene relación con las diatomeas y cianofitas, en el sentido de que en aguas eutróficas tienden a encontrar condiciones para su desarrollo.

Los Dinophyta y Chlorophyta son los grupos escasamente representados, posiblemente por las condiciones físico-químicas del medio.

En lo referente al análisis químico del cuerpo de agua, los resultados indican que se trata de aguas duras, cargadas de nutrientes que pueden servir para la crianza de diversos peces, especialmente trucha por su facil adaptabilidad a estos tipos de medios.

\section{BIBLIOGRAFÍA}

Fernandez, A. y Aguado, H. 1987. Botánica Criptogámica. Ed. U.N.T. Trujillo-Perú.

Franco, L. y Col. 1992. Fitoplancton del Río Caplina. Rev. Ciencia y Desarrollo No. 2. U.N.J.B.G. Tacna.

Gonzales de Infantes. 1988. El Plancton de las Aguas Continentales. Ed. Secretaria General de los Estados Americanos: Programa Regional de Desarrollo Científico y Tecnológico. Washington USA.

Morales, L. y Cadima, M. 1994. Estudio Cualitativo del Fitoplancton de 6 estanques de la Estación Piscícola Pirahiba-Cochabamba. Bolivia. Ed. Libro Resumen. XI. CONABIOL.

Coronel, N. 1994. Aspectos Limnológicos y Piscicolas de la laguna Aricota-Tacna. Ed. Libro Resumen XI. CONABIOL.

Manguin, M. 1996. Contribución al Conocimiento de las Diatomeas de los Andes del Perú. Memorias del Museo Nacional de Historia Natural Alemania.

Miranda, C. y Col. 1987. Contribución al Conocimiento dsel Fitoplancton del Lago Titicaca. Proyecto Evaluación de los Recursoos Pesqueros: Area Fitoplancton. Instituto de Ecologia-UMSA. La Paz, Bolivia.

Montoya, H. y Benavente, M. 1993 Microalgas Altoandinas de la localidad de Racura, Lima. Ed. Rev. Arnaldoa. Vol 1(4): 23-72. Trujillo. 


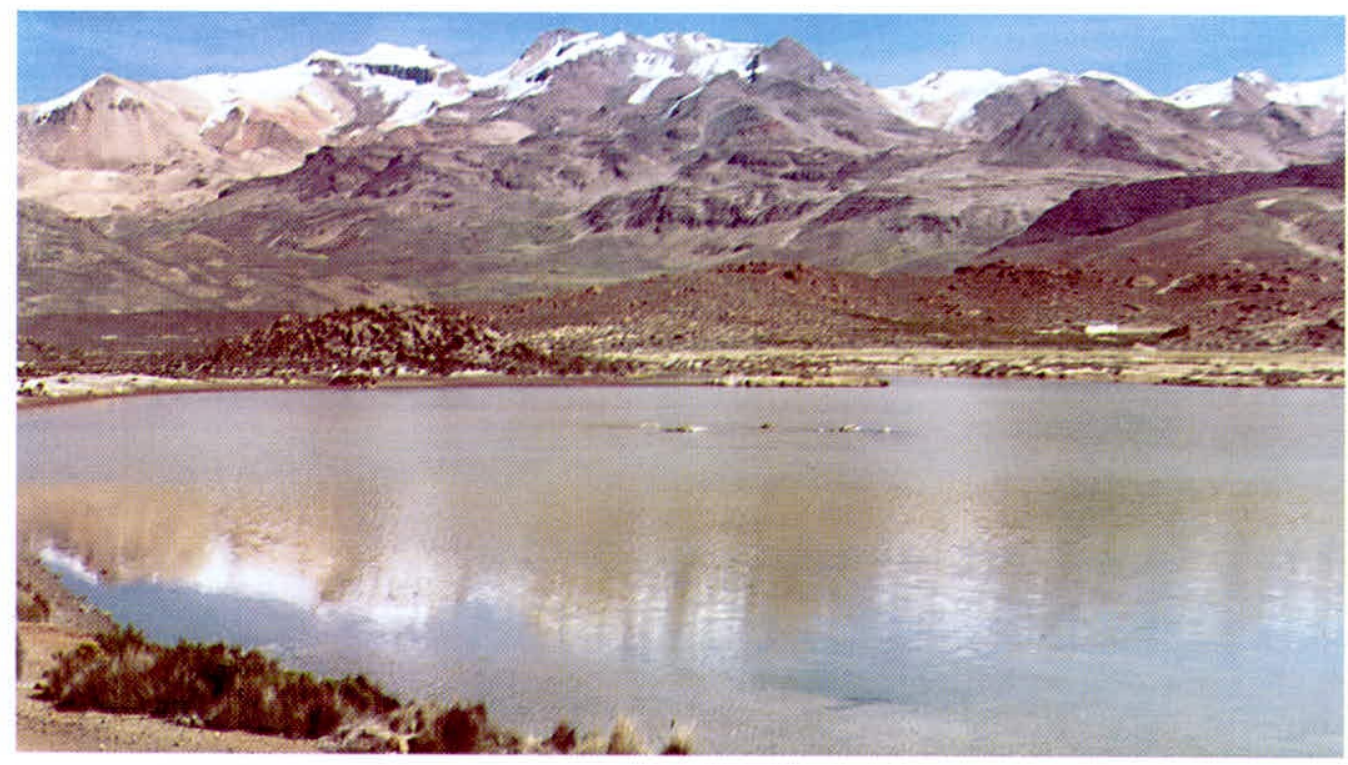

Laguna Paucarani - Tacna 4,135 m.s.n.m.

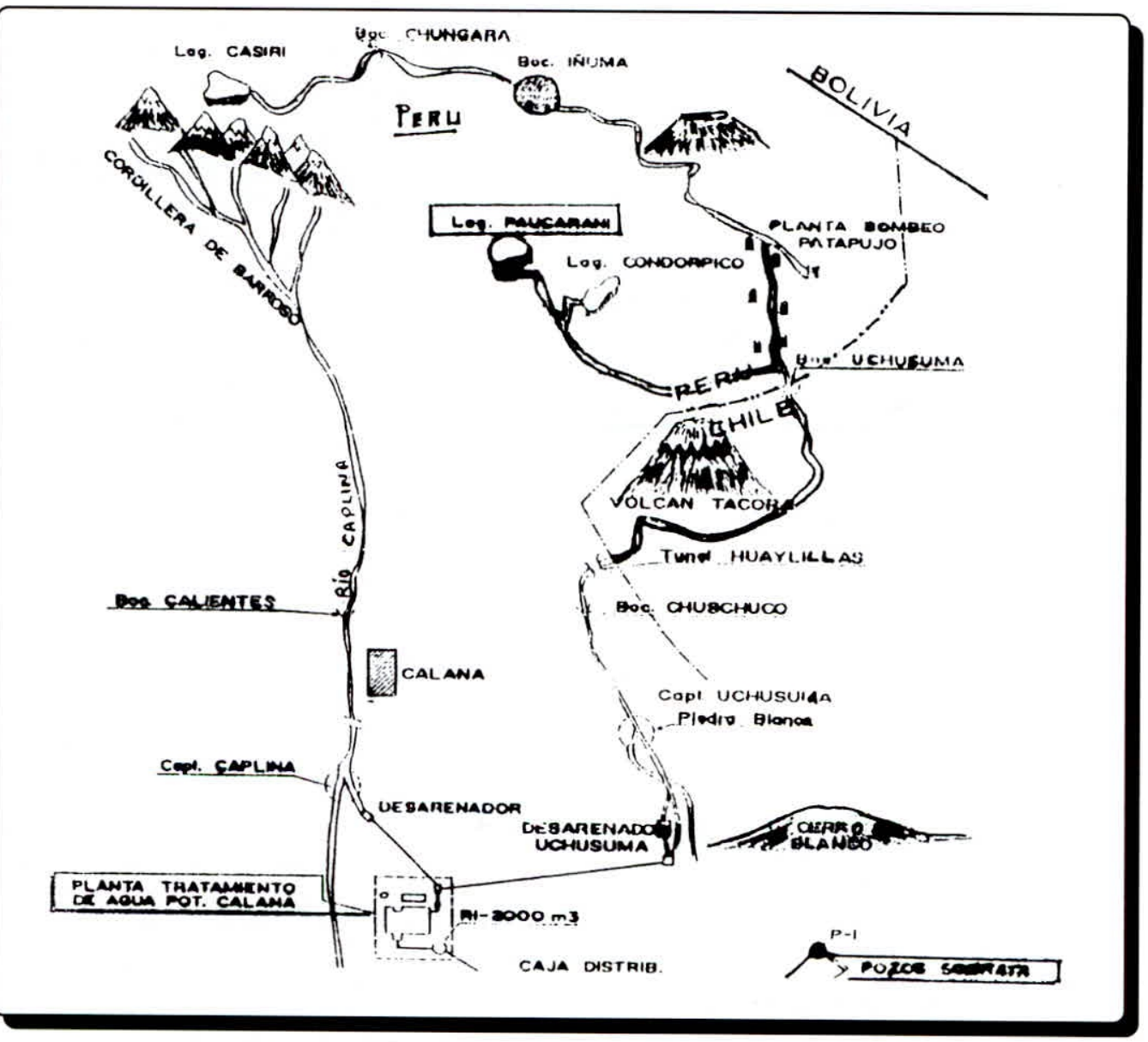

Ubicación geográfica de la laguna Paucarani. 


\section{FIGURA}

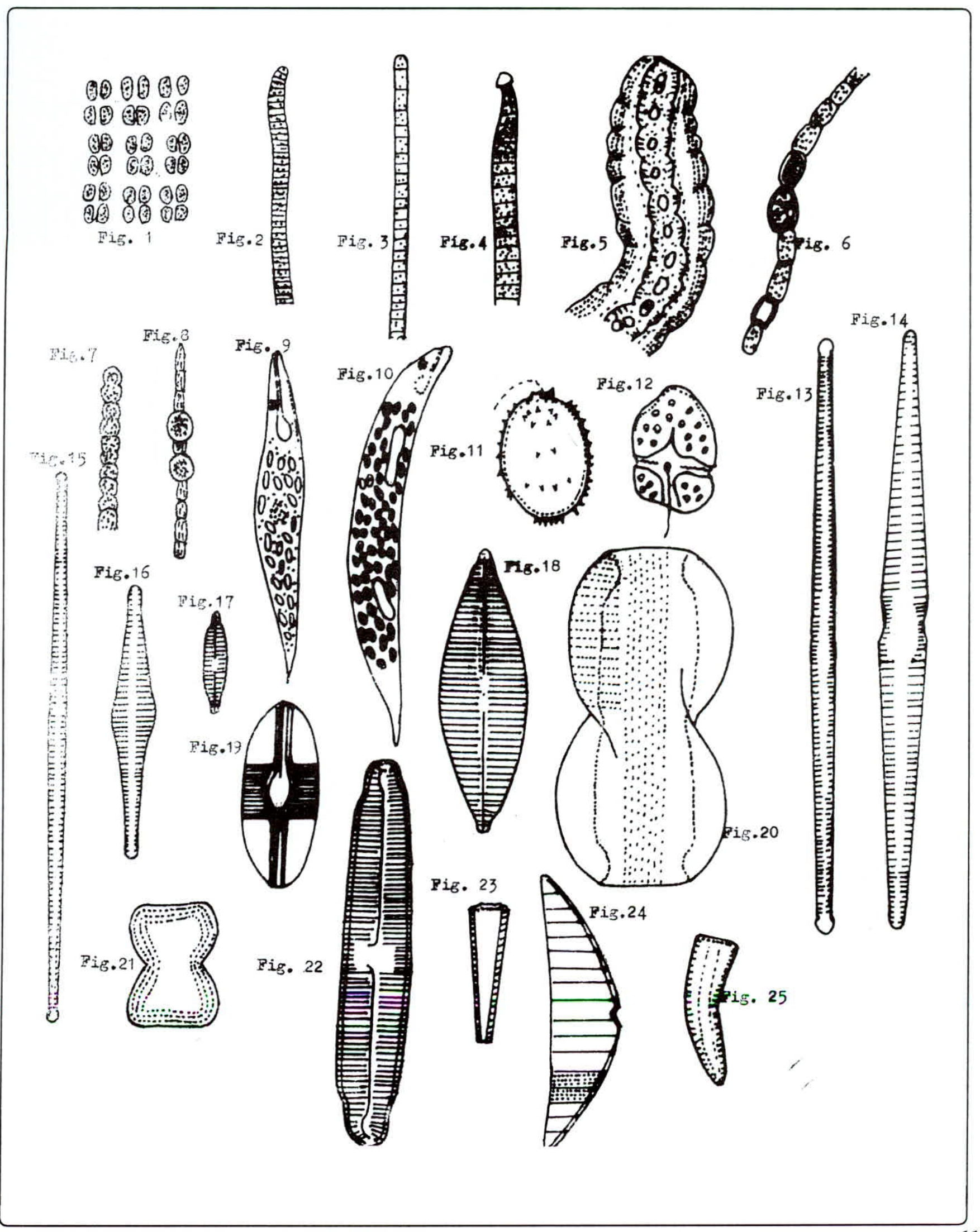




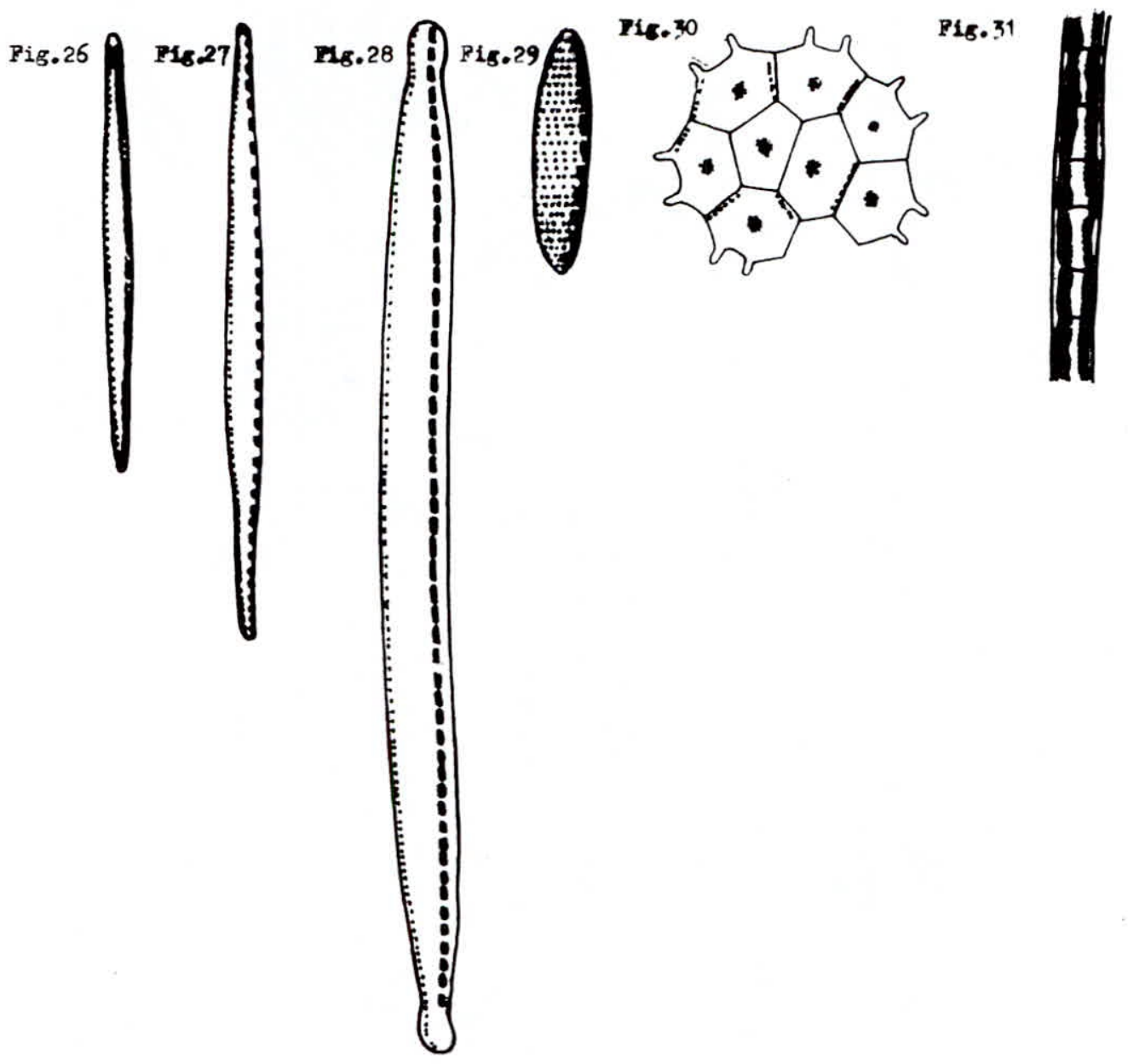

